\title{
Effect of Green Tea on Serum Cholesterol Level in Cholesterol Fed Rabbits.
}

\author{
Mohammad Aftabuddin ${ }^{1}$, Mohammed Irfan ${ }^{2}$, Omar Sadeque Khan ${ }^{3}$, Khan Mohammad Amanur Rahman ${ }^{4}$, Md. Rezwanul \\ Hoque $^{5}$, Nadira Islam ${ }^{6}$, Asit Baran Adhikary ${ }^{7}$ \\ ${ }^{1}$ Professor and Chairman, Cardiac Surgery, ${ }^{2}$ MS Student Cardivascular and Thoracic Surgery, ${ }^{3}$ Medical Officer, Cardiac Surgery, ${ }^{4}$ Medical Officer, \\ Cardiac Surgery, ${ }^{5}$ Associate Professor, Cardiac Surgery, ${ }^{6}$ Professor of Physiology, ${ }^{7}$ Professor of Cardiac Surgery, BSMMU
}

\begin{abstract}
:
Background: Since the development of atherosclerosis involves dyslipidemia, we postulated that green tea may exert lowering of serum cholesterol levels. Objectives: To investigate the effects of green tea on serum cholesterol level. Methods: Total 45 rabbits ( 15 were fed on $1 \%$ cholesterol and green tea for 16 weeks, another 15 were fed on $1 \%$ cholesterol and plain water for 16 weeks, last batch of 15 rabbits received normal rabbit diet for 16 weeks) in the department of Cardiac Surgery, Bangabandhu Sheikh Mujib Medical University, Dhaka from April 2011 to September 2012. They were evaluated for lipid profile and presence of atherosclerosis. Results: The mean total cholesterol in cholesterol fed rabbits with green tea after 16 weeks was $21.06 \pm 5.55$, LDL-C was $20.80 \pm 6.16$, Serum TG was $153.67 \pm 33.59$, HDL-C neither increased nor decreased. The mean total cholesterol in cholesterol fed rabbits with plain water after 16 weeks was 27.53 \pm 5.95 , LDL-C was $32.40 \pm 8.24$ and TG was $290.13 \pm 44.31$, no effect were seen on HDL-C. These rabbits were fed on green tea for 8 weeks, cholesterol levels returned to levels at commencement of experiment. Rabbits fed on normal rabbit diet for 16 weeks had TC $19.20 \pm 4.85$, LDL-C was $19.20 \pm 2.04$ and TG was $155.06 \pm 32.03 \mathrm{mg} / \mathrm{dl}$. HDL-C remained in normal range. But there was no atherosclerotic change in any group. Conclusion: Green tea significantly reduces serum cholesterol levels in cholesterol fed rabbits. There was no effect of green tea on HDL-C in any group.
\end{abstract}

Keywords: Green tea, cholesterol level, cholesterol fed rabbit.

[BSMMU J $2014 ; 7$ (2) : 91-94]

\section{Introduction:}

Tea is a product made from leaf and bud of the plant camellia sinensis which is the second most common consumed beverage in the world, well ahead of coffee, beer, wine and carbonated soft drink. ${ }^{1}$ With a per capita worldwide consumption of $0.12 \mathrm{~L} /$ day, habitual tea drinking has long been associated with health benefits and approximately 2.5 million metric tons of dried tea is produced each year, mainly India, China, Srilanka, Bangladesh, Turkey, Russia and Japan. The human use of camellia sinensis believed to have originated 4000 to 6000 years ago and it is generally regarded as safe by the US "Food and Drug Administration." Approximate 76-78 percent of the tea produced and consumed worldwide is black tea, 20-22 percent is green tea and 2 percent is oolong tea. ${ }^{2}$ Green tea is mainly consumed in Asia. ${ }^{2,3,4}$

Address for Correspondence: Professor Dr. Mohammad Aftabuddin Room No. 1203, D-Block, Department of Cardiac Surgery, BSMMU Phone: 88-02-9661438, E-mail: aftab12uddin@yahoo.com
Depending on the manufacturing process, teas are classified into: the non fermented green tea, the semi fermented oolong tea, and the fermented black tea. The chemical composition of tea is complex: polyphenols, catechins, caffeine, amino acids, carbohydrates, protein, chlorophyll, volatile compounds, fluoride, minerals and other undefined compounds. ${ }^{5}$ Among these, the polyphenols and catechins constitute the most interesting group of tea leaf components. ${ }^{5,6}$ The oxidized poly phenols are often called tannins, which is very different chemically from the commercial tannic acid of plant tannins. ${ }^{7}$ Green tea is a "non-fermented" tea and contains more catechins than black tea or oolong tea which is very strong antioxidants. ${ }^{8,9}$ Recent study suggests that green tea may contribute a role to reduce the risk cardiovascular. ${ }^{10,11,12,13,14}$ Atherosclerotic process, pathophysiological and biochemical background is similar like human, only difference is in rabbit the atherosclerotic process is very rapid. ${ }^{13,14}$ In recent years, the general popu- 
demonstrated increased awareness and interest in "Functional foods" with positive human health benefits. This study was designed, with a view to investigate the association between consumption of green tea and preventive effects of green tea against cardio vascular diseases by influencing the serum cholesterol, HDL-C, LDL-C and triglyceride levels in animal models.

Methods: This experimental study was conducted in the department of cardiac surgery, Bangabandhu Sheikh Mujib Medical University from April-2011 to December-2012. Total 45 locally bred white rabbits of male sex, weighing 1000 grams aged 6-9 months were housed individually under standard condition for a period of 2 weeks for acclimatization before commencing the experiments. Rabbits were randomized, 15 in group A-fed on $1 \%$ cholesterol and green tea for 16 weeks, 15 in group B-fed on $1 \%$ cholesterol and tap water for 16 weeks followed by green tea for 8 wks and last 15 rabbits in group $\mathrm{C}$-were fed on normal rabbit diet for 16 weeks. Hypercholesterolemic diet containing $1 \%$ cholesterol which is readily available in the market was added to the normal diet of rabbits. Dried leaves of green tea locally produced was mounted on a $90^{\circ} \mathrm{C}$ tap water for 5 minutes and the filtrate was left to come at room temperature until provided to experimental group. Hypercholesterolemic feed was restricted to 80 gram/day to experimental animals, but drinking water or water boiled with green tea was freely available. Overnight fasting blood samples were collected for estimation of lipids from all animals at the commencement of the experiment once every four weeks from their lateral ear veins and from aorta at the end of experiment after sacrifice. The serum was separated and stored at $-20^{\circ} \mathrm{C}$ until estimation. At the end of the 16 weeks period the animals in group-A and group-C were sacrificed, at the end of 24 weeks, animals in group B were sacrificed. Part of the aorta and coronary arteries were taken for detection of atherosclerosis. Three to four pieces of more or equal length were opened longitudinally and fixed flatly in $10 \%$ phosphate buffered neutral formalin. Statistical analysis was conducted using SPSS 12 for windows software. The quantitative values were expressed as mean $\pm \mathrm{SD}$; paired t-test was done for analysis. $\mathrm{P}$ value of $<0.05$ was considered significant.

\section{Results:}

Total 45 rabbits, 15 in each group were studied. In group A, Green tea reduced total cholesterol, LDL-C and triglyceride levels in rabbit fed with hypercholesterolemic diet containing $1 \%$ cholesterol and the result was statistically significant $(p<0.05)$. But green tea marginally increased HDL-C which is not statistically significant $(\mathrm{P}>0.05)$.

In Group B, serum total cholesterol, LDL-C and triglyceride levels increased in rabbits fed with hypercholesterolemic diet containing $1 \%$ cholesterol and plain tap water and result was statistically significant $(\mathrm{p}<0.05)$. However HDL-C did not show any significant change $(\mathrm{p}>0.05)$. Further this group received green tea for 8 weeks, serum total cholesterol, HDL-C, LDL-C and triglyceride levels approximately returned to levels at the commencement of the experiment.

In group $\mathrm{C}$, rabbits fed with normal diet consisting of green leafy vegetables, lipid profile both before the starting of experiment and at the end of 16 weeks remained within normal levels and the difference was not statistically significant $(p>0.05)$. Above biochemical results are shown in the table No-1.

Histopathological study for atherosclerotic lesion after staining with haematoxylin-eosin for microscopic examination were done. But atherosclerotic lesion was not detected in any group.

\section{Discussion:}

As mentioned above, the laboratory studies have indicated that green tea may have a protective effect against the rise of serum total cholesterol, LDL-C and triglyceride which is presented in the table-I among the group A and group B. 
Our result is in consistent with some other studies in experimental animals. ${ }^{13,14,15,16,17,18}$ Some others human study shows similar finding like our experimental research study. ${ }^{19,20,21,22,23,24}$

In this study there are no marked influence of green tea on HDL-C level in any group which is also supported by similar type study..$^{13,14,15,16,17,18}$ There was no influence on HDL-C in any group. There was no histological feature of atherosclerosis on aorta or on coronary artery which has difference with some other researcher. ${ }^{13}$ This difference may be due to short duration of our study period where others study duration was more than this study. A significant time may be required to develop the atherosclerosis in aorta and coronary artery which may possible to detect by histological study. In our study, we have not done any immunohistochemical study to observe the early onset of atherosclerotic process which can be detected by using mouse monoclonal anti-human vascular endothelial growth factor (VEGF) antibody.

Although there has been a substantial amount of research on teas and their health benefits, it is difficult to compare in vitro and in vivo data across laboratories due to lack of standardized experimental procedure.

From the result of present study it is obvious that the administration of green tea in rabbit fed with hypercholesterolemic diet has a lowering effect on the lipid profile. As beneficial effects of green tea are being increasingly proved it may be advised to encourage the regular consumption of this widely available, tasty and inexpensive beverage as an interesting alternative to other drinks. We are in favor of prolonging the duration of experiment and also including more animal subjects.

\section{References :}

1. Costa LM, Gouveia ST, Nobrega JA: Comparison of heating extraction procedures for $\mathrm{Al}, \mathrm{Ca}, \mathrm{Mg}$ and $\mathrm{Mn}$ in tea samples. Ann Sci 2002; 18:313-318.

2. Gour S, Agnihotri R, Greea te: A novel functional food for the oral health of older adults. geriatrics \& Gerontology intarantional al $2014 ; 14: 238-250$
3. Zuo Y, Chen H and Deng Y. Simultaneous determination of catechins, caffeine and gallic acids in green, Oolong, black and Pu-erh teas using HPLC with a photodiode array detector.Talanta.2002; 57:307-316.

4. Ilyasova D, Martin C and Sandier RS. Tea intake and risk of colon cancer in African- Americans and Whites: North Carolina colon cancer study. Cancer Causes Control. 2003; 14:676-772.

5. Graham HN. Green tea composition, consumption, and polyphenol chemistry. Prev Med.1992; 21:334-350.

6. Mitscher LA, Jung M, Shankel D, Dou JH, Steele L, Pillai SP Chemoprotection: a review of the potential therapeutic antioxidant properties of green tea (Camellia sinensis) and certain of its constituents. Med Res Rev. 1997; 17:327-365.

7. Wheeler S. Tea and tannins. Science. 1979; 204:6-8

8. Wu CD and Wei G X. Tea as a functional food for oral health. Nutrition. 2002; 18: 443-444.

9. Kuriyama S. Green tea consumption and prevention of coronary artery disease. Circ J. 2010; 74:248-249.

10. Peters U, Poole C and Arab L. Does tea affect cardiovascular disease? A meta-analysis. Am J Epidemiol. 2001; 154:495-503.

11. Tomita T, Miura Y, Chiba T, Kawai E, Umegaki K, Miura S et al. Antiatherogenic effects of tea polyphenols (flavan-3-ols) in humans and apoE-deficient mice. Basic Life Sci. 1999; 66: $471-482$.

12. Ivanov V, Roomi MW, Kalinovsky T, Niedzwiecki A and Rath M. Anti-atherogenic effects of a mixture of ascorbic acid, lysine, praline, arginine and green tea phenols in human aortic smooth muscle cell. 12th International Congress on Cardiovascular Pharmacotherapy, Barcelona, Spain, 2003; May 7-10.

13. Kavantzas N, Chatziioannou A, Yanni AE, Tsakayannis D, Alafoutas D, Agrogiannis G et al. Effect of green tea on angiogenesis and severity of atherosclerosis in cholesterol-fed rabbit. Vascul Pharmacol. 2006; 44: 461-463.

14. Tijburg LBM, Wiseman SA, Meijer GW and Weststrate JA. Effects of green tea, black tea and dietary lipophylic antioxidants on LDL oxidizability and atherosclerosis in hypercholesterolemic rabbits. Atherosclerosis. 1997; 135:37-47.

15. Suzuki Y, Unno T, Kobayashi M, Nozawa A, Sagesaka Y and Kakuda T. Dose- dependent suppression of tea catechins with a galloyl moiety on postprandial hypertriglyceridemia in rats. Biosci Biotechnol Biochem. 2005; 69: 1288-1291.

16. Babu PV, Sabitha KE and Shyamaladevi CS. Green tea extract impedes dyslipidaemia and development of cardiac dysfunction in streptozotocin-diabetic rats. Clin Exp Pharmacol Physiol. 2006; 33:1184-1189.

17. Yang $\mathrm{M}$, Wang $\mathrm{C}$ and Chen $\mathrm{H}$. Green, oolong and black tea extracts modulate lipid metabolism in hyperlipidemia rats fed high sucrose diet. J Nutr Biochem. 2001; 12: 14-20.

18. Hasegawa N, Yamda N and Mori M. Powdered green tea has antilipogenic effect on Zucker rats fed a high-fat diet. Phytother Res. 2003; 17: 477-480. 
19. Johanna $\mathrm{M}$, Geleijnse, $\mathrm{PhD}$, Lenore $\mathrm{J}$, Launer $\mathrm{PhD}$, Albert Hofman MD, Huibert AP, et al. Tea Flavonoids May Protect Against Atherosclerosis: The Rotterdam Study. Arch Intern Med. $1999 ; 159: 2170-2174$

20. Sano J, Inami S, Seimiya K, Ohba T, Sakai S, Takano T and Mizuno KJ. Effects of green tea intake on the development of coronary artery disease. Circ J. 2004; 68:665-670.

21. Imai $\mathrm{K}$ and Nakachi $\mathrm{K}$. Cross sectional study of effects of drinking green tea on cardiovascular and liver diseases. BMJ. 1995; 310: 693-696.

22. Sasazuki S, Kodama H, Yoshimasu K, Liu Y, Washio M and
Tanaka K. Relation between green tea consumption and the severity of coronary atherosclerosis among Japanese men and women. Ann Epidemiol. 2000; 10: 401-408.

23. Erba D, Riso P, Bordoni A, Foti P, Biagi PL and Testolin G. Effectiveness of moderate green tea consumption on antioxidative status and plasma lipid profile in humans. J Nutr Biochem. 2005; 16: $144-149$

24. Unno T, Tago M, Suzuki Y, Nozawa A, Sagesaka YM, Kakuda T, et al. Effect of tea catechins on postprandial plasma lipid responses in human subjects. Br J Nutr. 2005; 93:543-547. 